\title{
JHU-2545 Selectively Shields Salivary Glands and Kidneys during PSMA- Targeted Radiotherapy
}

Michael T. Nedelcovych ${ }^{1,2}$, Ranjeet P. Dash ${ }^{1,2}$, Ying Wu ${ }^{1}$, Eun Yong Choi ${ }^{8}$, Rena S. Lapidus ${ }^{8}$, Pavel Majer ${ }^{9}$, Diane Abou ${ }^{11}$, Marie-France Penet ${ }^{4,7}$, Anastasia Nikolopoulou ${ }^{10}$, Alex AmorCoarasa $^{10}$, John Babich ${ }^{10}$, Daniel L. Thorek ${ }^{11}$, Rana Rais ${ }^{1,2}$, Clemens Kratochwil ${ }^{12}$, Barbara S. Slusher ${ }^{1,2,3,4,5,6^{*}}$

${ }^{1}$ Johns Hopkins Drug Discovery, Departments of ${ }^{2}$ Neurology, ${ }^{3}$ Medicine, ${ }^{4}$ Oncology,

${ }^{5}$ Psychiatry, ${ }^{6}$ Neuroscience, ${ }^{7}$ Radiology and Radiological Science, Johns Hopkins School of Medicine, Baltimore, MD 21205, U.S.A.

${ }^{8}$ Translational Laboratory Shared Service, University of Maryland School of Medicine, 655 West Baltimore Street, Baltimore, MD, 21201, U.S.A.

${ }^{9}$ Institute of Organic Chemistry and Biochemistry, Academy of Sciences of the Czech Republic v.v.i., Prague, 166 10, Czech Republic

${ }^{10}$ Division of Radiopharmaceutical Sciences and MI(3), Department of Radiology, Weill Cornell Medicine, New York, NY, U.S.A.

${ }^{11}$ Depatment of Radiology, Washington University School of Medicine, Saint Louis, MO, 63110, U.S.A.

${ }^{12}$ Department of Nuclear Medicine, University Hospital Heidelberg, Heidelberg, Germany

\section{Address Correspondence to:}

*Barbara S. Slusher, PhD, Johns Hopkins Drug Discovery, 855 North Wolfe Street, Baltimore, Maryland, USA 21205, Phone: 410-614-0662, Fax: 410-614-0659, E-mail: bslusher@jhmi.edu

This manuscript has been withdrawn by the authors because data included in the original manuscript will be separated into two different manuscripts and submitted for publication to two different journals. The authors do not wish this work to be cited as reference for the project. If you have any questions, please contact the corresponding author. 